\title{
Tuning the growth of metal-organic framework nanocrystals by using polyoxometalates as coordination modulators
}

\author{
Xiaobin Xu, Yan Lu, Yong Yang, Farhat Nosheen and Xun Wang*
}

Host-guest supramolecular interactions, which exist widely between diverse metal cations and polyoxometalate (POM) anions, have always been utilized to construct metal-organic frameworks (MOF) /POMs bulk composites. Actually, the interactions can also be found application in realizing shape-controlled synthesis of MOF nanocrystals (NCs). In this context, simply by introducing Keggin-type POMs into MOF precursors, various uniform $\left[\mathrm{Cu}_{3}(\mathrm{BTC})_{2}\right]_{n}(\mathrm{BTC}=$ benzene-1,3,5-tricarboxylate) NCs, including (truncated) octahedrons, hollow hierarchical spheres and octahedrons, are fabricated. Besides, such reaction system can be applied to construct noble metal@ MOF nanocomposite, which significantly enhances the catalytic performance of the noble metal.

\section{INTRODUCTION}

Metal-organic frameworks (MOFs) are a kind of crystalline porous assemblies of metal ions (or metal clusters) and organic ligands linked through coordination bonds [1] and exhibit outstanding properties [2-7]. Downsizing of MOF crystals into nano- or micrometer regime is of great significance [8-11], because traditional bulk crystalline materials do not always fulfill the specific needs for these applications, such as smart membranes [12], thin films devices [13] and drug delivery [14-16]. In order to optimize the properties for specific applications, manipulation of the shape and structure (solid vs. hollow), including creating more novel functional structures/shapes, is still urgently in need [17-19]. This goal may be fulfilled by adopting an effective additive to modulate the nucleation and growth processes of MOF crystals [20-23].

Polyoxometalates (POMs) are a class of inorganic clusters of the early transition elements and have numerous intriguing properties [24-26]. Generally, POMs act as anionic templates/structure directing agents to build POM-based MOF bulk composites through the host-guest supramolecular interactions (such as weak coordination interactions, electrostatic interaction, and hydrogen bonding) [20,27]. That is, POMs affect the nucleation and growth processes of MOFs. Based on the above facts, we wondered whether POM could be used as coordination modulator to synthesize nanoscale MOFs. When POMs were added into the precursor solution for preparing MOF crystals, the hostguest supramolecular interactions between POMs and MOFs would alter the equilibrium between the metal cations and the organic ligands and affect the situation of the reactants, thus controlling the synthesis of MOFs.

In order to demonstrate the feasibility of this method, Keggin-type POM (sodium phosphotungstate, abbreviated as NaPW) assisting synthesis of $\left[\mathrm{Cu}_{3}(\mathrm{BTC})_{2}\right]_{n}\left(\mathrm{BTC}^{3-}=\right.$ benzene-1,3,5-tricarboxylate) is selected as an example.

$\left[\mathrm{Cu}_{3}(\mathrm{BTC})_{2}\right]_{n}$, also known as HKUST-1, is a representative MOF material first reported by Chui et al. [28]. The $\left[\mathrm{Cu}_{3}(\mathrm{BTC})_{2}\right]_{n}$ nanocrystals were prepared by mixing POM (NaPW) aqueous solution with the reaction solution containing MOF precursors [Cupric acetylacetonate and $\mathrm{H}_{3} \mathrm{BTC}$, $\mathrm{N}, \mathrm{N}$-dimethylformamide (DMF) and polyvinylpyrrolidone (PVP), followed by microwave irradiation at $373 \mathrm{~K}$ for $10 \mathrm{~min}$. Scheme 1 shows the TEM/SEM images of $\left[\mathrm{Cu}_{3}(\mathrm{BTC})_{2}\right]_{n}$ uniform nanocrystals with diverse morphologies. The typical quantities of NaPW and water used in the reaction medium are listed in Table S1.

\section{EXPERIMENTAL METHODS}

\section{Preparation of $\left[\mathrm{Cu}_{3}(\mathrm{BTC})_{2}\right]_{n}$ Crystal of different morphological types}

Stock solutions were prepared by dissolving $1 \mathrm{~g}$ 1,3,5-benzenetricarboxylic acid $\left(\mathrm{H}_{3} \mathrm{BTC}\right)$ and $23.775 \mathrm{~g}$ lauric acid in $50 \mathrm{~mL}$ butanol respectively. $\mathrm{NaPW}\left(\mathrm{Na}_{2} \mathrm{H}_{5} \mathrm{PWO}_{42}\right)$ was purchased from Sinopharm Chemical Reagent Co, Ltd. $\mathrm{NaPW}$ aqueous solution was prepared by dissolving NaPW solid in certain amount of distilled water. Cupric acetylacetonate $\left(\mathrm{Cu}(\mathrm{acac})_{2}, 22.2 \mathrm{mg}, 0.0845 \mathrm{mmol}\right)$ was dissolved in $8 \mathrm{~mL}$ DMF by ultrasonic treatment. Then the NaPW aqueous solution, $0.5 \mathrm{~mL}$ lauric acid solution and $0.5 \mathrm{~mL}$ BTC solution were added successively into the DMF solu- 


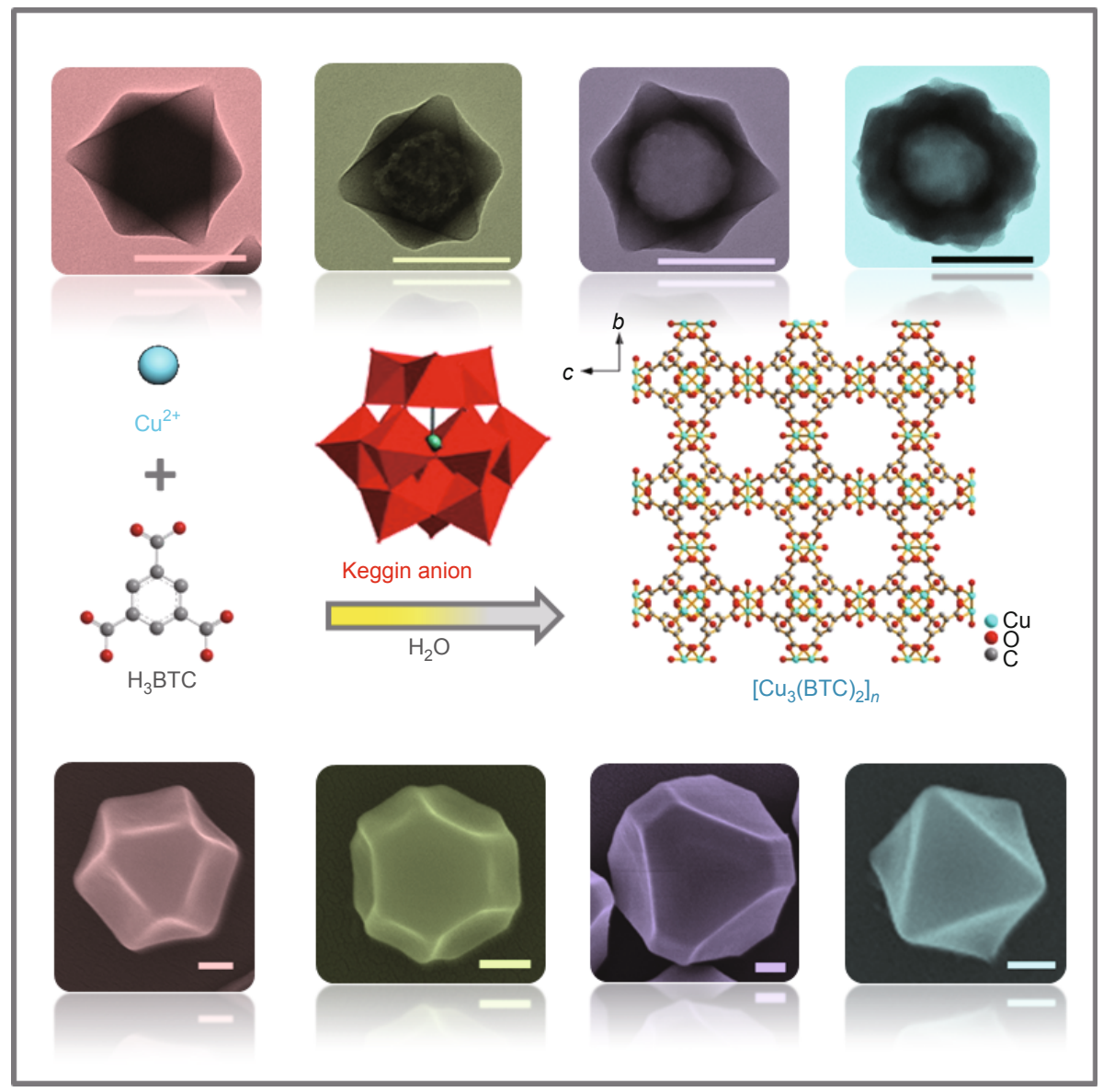

Scheme 1 Schematic illustration of the preparation of $\left[\mathrm{Cu}_{3}(\mathrm{BTC})_{2}\right]_{n}$ nanocrystals modulated by POM and water. At the top and bottom of the scheme: representative TEM/SEM images of the uniform $\left[\mathrm{Cu}_{3}(\mathrm{BTC})_{2}\right]_{n}$ crystals prepared in this work (scale bar is $200 \mathrm{~nm}$ ).

tion. After stirring for several minutes, the starting solution was transferred into a $20 \mathrm{~mL}$ Pyrex microwave vial. The mixture was heated by microwave irradiation (Initiator 2.5 microwave from Biotage) at $353 \mathrm{~K}$ for $10 \mathrm{~min}$. The resulting blue powder was isolated by centrifugation and washed twice with DMF and ethanol. See supplementary information (SI) for additional experimental details.

\section{RESULTS AND DISCUSSION}

\section{Morphogenesis modulated by POM anions}

Firstly, the amount of POMs added in the reaction medium had a significant effect on the morphology of the final MOF crystals. Without or with low amount of NaPW (for example, $8.1 \mathrm{mg}$ ), ill-defined worm-like particles (Fig. S1) were obtained. Interestingly, when the amount of POM was increased to $13.5 \mathrm{mg}$, the morphology was transformed into uniform nanooctahedrons (type i, Figs $1 \mathrm{a}, 1 \mathrm{~b}$ and S2). Type i crystals was pure-phase $\left[\mathrm{Cu}_{3}(\mathrm{BTC})_{2}\right]_{n}$, which was confirmed by both powder X-ray diffraction (PXRD) pattern (Fig. S3) and FT-IR spectra (Fig. S4). The nitrogen adsorption-desorption isotherms and pore size distribution measurements (Fig. S5) and Thermogravimetric (TG) analysis (Fig. S6) were also taken.

In order to investigate the underlying mechanism, the supramolecular interactions between the metal and POM ions as well as their influence on the nucleation-growth processes were studied. Direct evidence of the interactions between $\mathrm{Cu}(\mathrm{II})$ and $\mathrm{POM}$ ions was obtained from ${ }^{31} \mathrm{P} \mathrm{NMR}$ spectra of solutions prepared from $\mathrm{Cu}\left(\mathrm{NO}_{3}\right)_{2} \cdot 3 \mathrm{H}_{2} \mathrm{O}$ and NaPW. Upon adding $\mathrm{Cu}(\mathrm{II})$ cations, the ${ }^{31} \mathrm{P}$ resonance of the POM anions shows a significant shift from -10.15 to -29.97 ppm (Fig. 1c), indicating the change of local environment of the central P atom in the POM anion [29]. Such impact is the result of the interaction with $\mathrm{Cu}$ (II) cations, and the peak broadening might be attributed to the intricately or randomly orienting of $\mathrm{Cu}$ (II) cations around the surface of POM anions. Substantial evidence was further obtained from the UV-vis spectroscopy measurements. The absorption maximum $\left(\lambda_{\max }\right)$ of DMF solution of $\mathrm{Cu}(\mathrm{acac})_{2}$ 
a
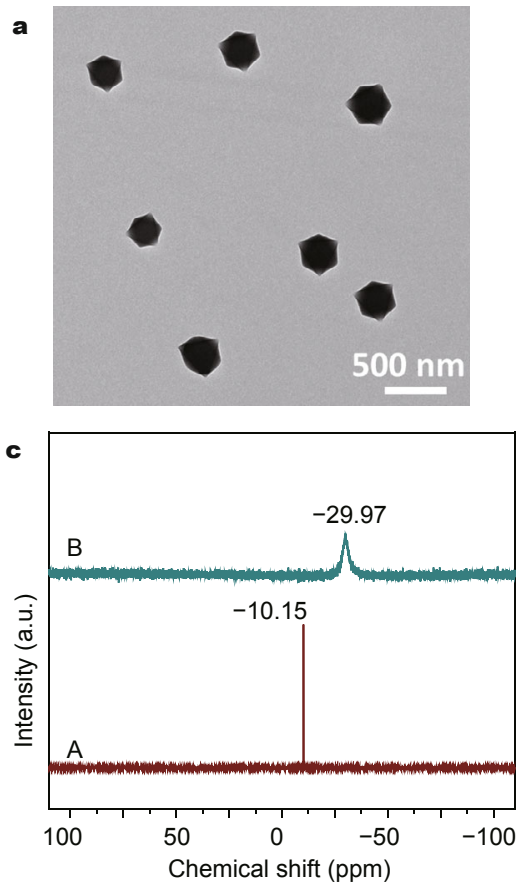

b
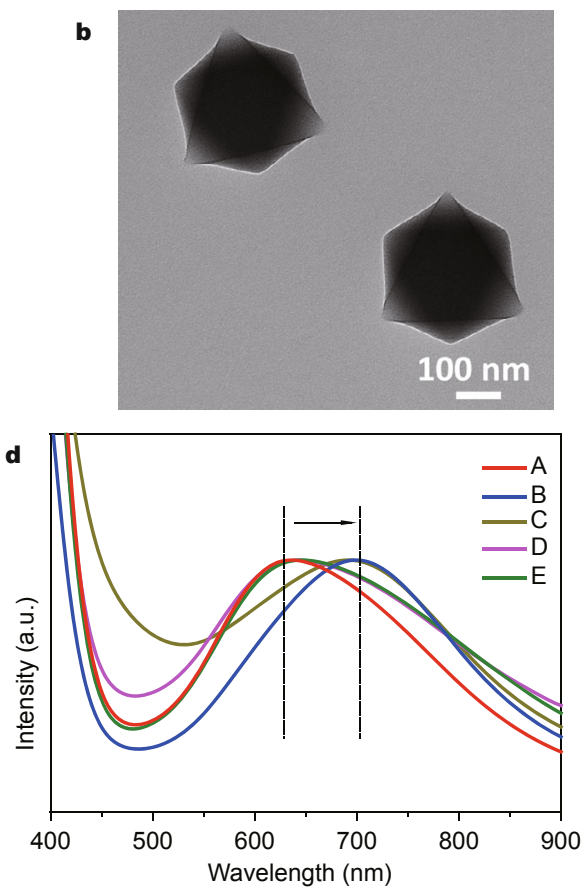

Figure 1 (a, b) TEM images of $\left[\mathrm{Cu}_{3}(\mathrm{BTC})_{2}\right]_{n}$ crystals of type i with $13.5 \mathrm{mg} \mathrm{NaPW}$ added. (c) ${ }^{31} \mathrm{P}$ NMR spectra of a solution of (A) NaPW; (B) a solution of $\mathrm{Cu}\left(\mathrm{NO}_{3}\right)_{2} \cdot 3 \mathrm{H}_{2} \mathrm{O}$ and NaPW. (d) UV-vis spectra of (A) DMF solution of $\mathrm{Cu}(\mathrm{acac})_{2}$; (B-E) (A) solutions with addition of $0.5 \mathrm{~mL} \mathrm{H}_{3} \mathrm{BTC}$ solution and different amount of NaPW, i.e., 0, 13.5, 27.0, 54.0 mg, respectively.

peaked at $635 \mathrm{~nm}$. In absence or with low amount of POM anions, upon addition of $\mathrm{H}_{3} \mathrm{BTC}, \lambda_{\max }$ red-shifts to $697 \mathrm{~nm}$ (Fig. 1d, lines B and C), indicating that the ligand-exchange reaction occurred and the dinuclear copper paddlewheel cluster formed [22]. However, with high amount of POM anions, $\lambda_{\max }$ does not shift after addition of ligands (Fig. 1d, lines $\mathrm{D}$ and $\mathrm{E}$ ). That is, the complexation between the metal source and organic ligands is inhibited due to the interactions between $\mathrm{Cu}(\mathrm{II})$ cations and POM anions.

The interactions could be further verified by the result of changing the adding sequence of the reactants: inverting the normal addition sequence of $\mathrm{NaPW}$ resulted in different morphologies. When $\mathrm{H}_{3} \mathrm{BTC}$ was added prior to $\mathrm{NaPW}$, and all other conditions were kept constant, wormlike structures interconnected by distorted octahedrons were obtained, as shown in Fig. S7, totally distinct from the uniform octahedron (type i).

Based on the experimental evidence, the role of POM in MOF growth can be described as follows: without or with low amount of POM, the existence of high population of free metal cations and organic ligands in the system leads to poor kinetic control, thus finally resulting in illdefined worm-like particles. When the amount of POM is increased to a proper amount, the separation of nucleation and growth processes could be achieved: after fast nucleation event, slow growth occurs owing to $\mathrm{Cu}(\mathrm{II})$ cations be- ing released slowly and smoothly, confirming that the rate of feedstock consumption by the growing crystals is not exceeded by the rate of metal cations production [30]. Thus no new nuclei forms and the nuclei will grow into uniform $\left[\mathrm{Cu}_{3}(\mathrm{BTC})_{2}\right]_{n}$ nanocrystals (octahedron, type i).

Further increasing the amount of POM, the morphologies of MOF can be greatly controlled from solid octahedral NCs with different truncated degree to even hollow cages. When the amount of NaPW was increased to 21.6 $\mathrm{mg}$ from $13.5 \mathrm{mg}$, the morphology turned into edge- and corner-rounded octahedrons (type ii, Figs $2 \mathrm{a}$ and b) from well-defined octahedrons (type i, Figs $1 \mathrm{a}$ and b), accompanied by partial hollowness. When the amount of NaPW reached $32.4 \mathrm{mg}$, the shape turned to hierarchical spheres with same partial hollowness (type iii, Figs $2 \mathrm{c}$ and d). As the amount of NaPW was increased to $37.8 \mathrm{mg}$, the hierarchical spheres became completely hollow (type iv, Figs 2e and $\mathrm{f}$ ), which could be clearly seen from the TEM (Fig. 2e) and SEM images (Fig. 2f, inset shows a broken particle). If the amount of $\mathrm{NaPW}$ was increased further to $54.0 \mathrm{mg}$, the resulting morphologies were partially broken hollow rough spheres (type v, Fig. S9). This result might be explained as follows: as the amount of POM was increased further, concentration of free metal cations reduced and the release of metal became more difficult. Therefore, the newly formed primary nanocrystals with small-size would minimize the 

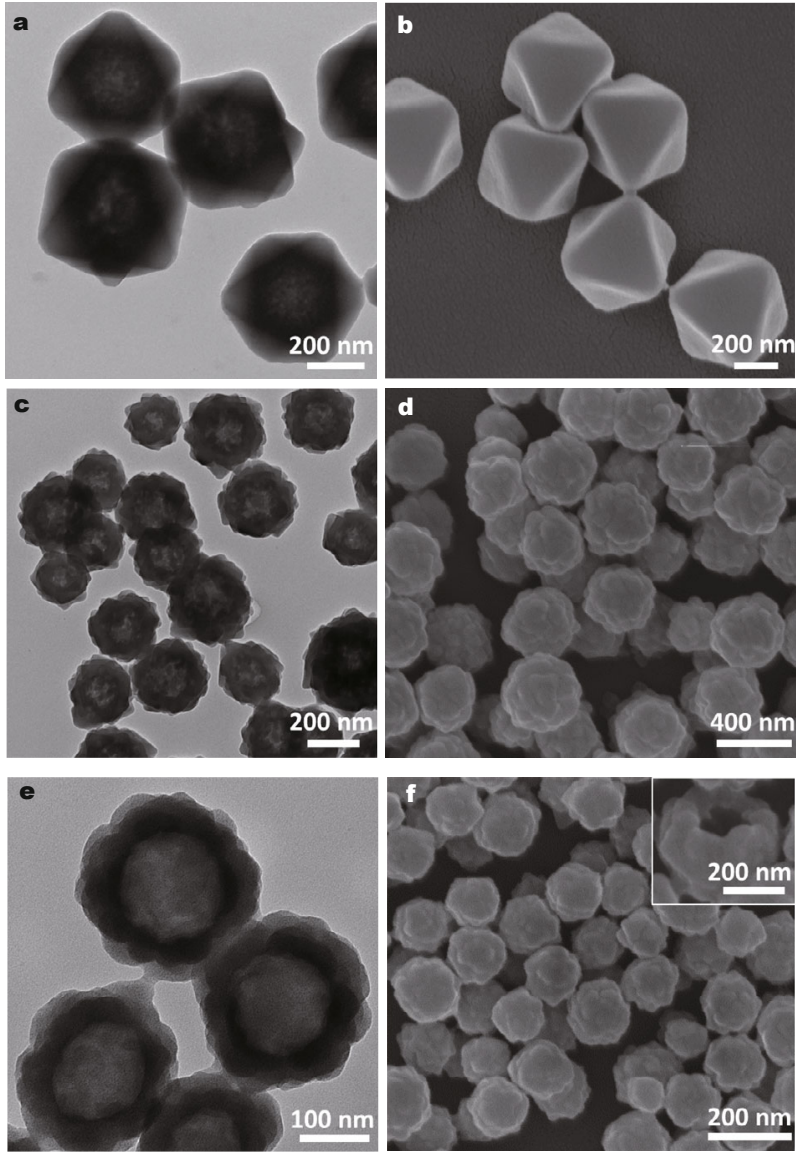

Figure 2 (a, c, e) TEM and (b, d, f) SEM images of $\left[\mathrm{Cu}_{3}(\mathrm{BTC})_{2}\right]_{n}$ crystals of (a, b) type ii, (c, d) iii, (e, f) iv, respectively.

surface energy via Ostwald ripening (see below).

\section{Control of the exposed faces of MOF nanocrystals}

In the above experiments, the role of POM was explored by keeping the volume of water constant. In fact, water was another important influencing factor. Keeping the quantities of POM identical and increasing the amount of water permitted the formation of truncated octahedron with exposed faces controllable. As shown in Figs $3 a$ and b, increasing the amount of water from $0.15 \mathrm{~mL}$ to $0.50 \mathrm{~mL}$ lead to a morphology change from type $\mathrm{v}$ to cubeoctahedron (type vi), with eight triangular faces and six square faces, being indexed to/as $\{111\}$ and $\{100\}$ facets, respectively. The area ratio of $\{111\}$ and $\{100\}$ facets was found to be $1: \sqrt{3}$, indicating dominance of $\{100\}$ facets in the structure. When the amount of water was increased to $0.6 \mathrm{~mL}$, the area of squares decreased and triangles got converted to regular hexagon, leading to the formation of truncated octahedron (type vii, Figs $3 \mathrm{c}$ and $\mathrm{d}$ ), with 8 regular hexagonal faces and 6 square faces, corresponding to $\{111\}$ and $\{100\}$
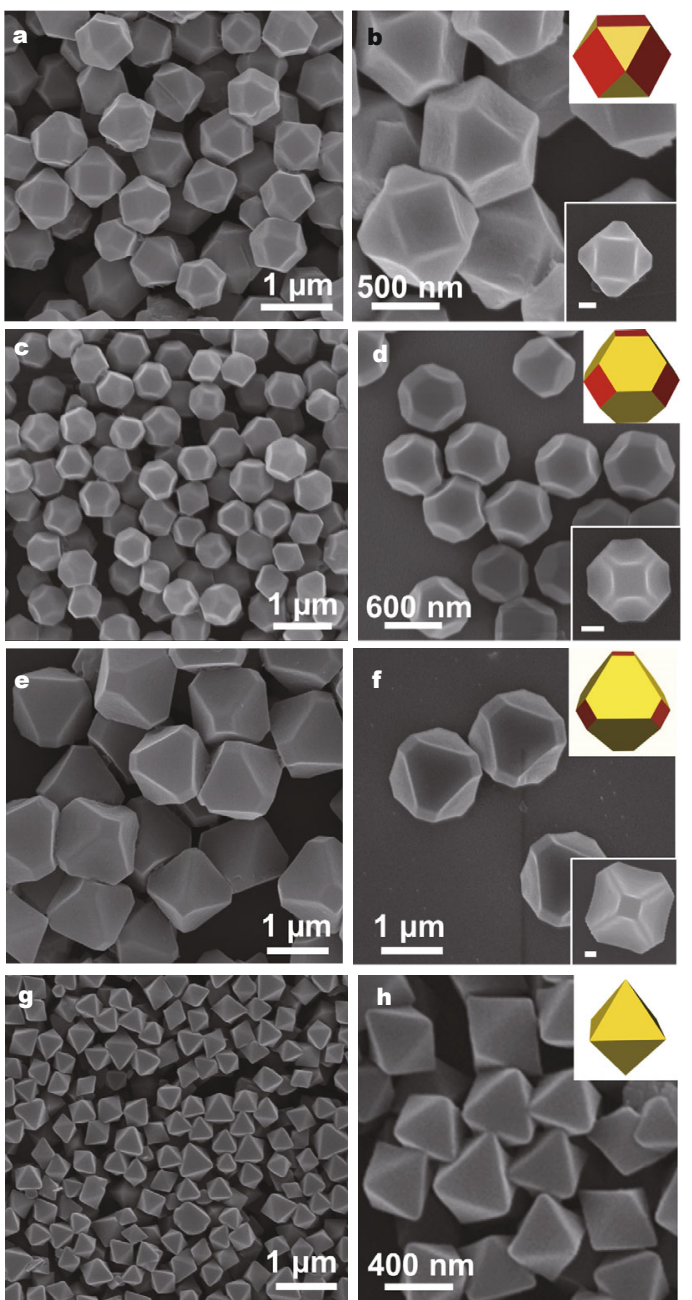

Figure $3(\mathrm{a}-\mathrm{h})$ SEM images of $\left[\mathrm{Cu}_{3}(\mathrm{BTC})_{2}\right]_{n}$ crystals of $(\mathrm{a}, \mathrm{b})$ type vi, $(c, d)$ vii, (e, f) viii and $(g, h)$ ix, respectively. Scale bars of insets in (b, $\mathrm{d}, \mathrm{f})$ are $200 \mathrm{~nm}$.

facets, respectively. Compared to the cubeoctahedron, the area ratio in $\{111\}$ and $\{100\}$ facets was $2 \sqrt{3}: 1$, indicating dominance of $\{111\}$ facets in the truncated octahedron. Upon further increasing the amount of water to $0.7 \mathrm{~mL}$, the square face got further squeezed, with shape turning to slightly truncated octahedron (type viii, Figs $3 e$ and $f$ ), in which the length ratio of the hexagon is $2.5: 1$. In the end, when $0.8 \mathrm{~mL}$ water was used, the shape totally converted to octahedron (type ix, Figs $3 g$ and h), with only $8\{111\}$ facets.

In fact, geometrical shape of cubooctahedral NCs is a function of the ratio, $R$, of the growth rate along the $\langle 100\rangle$ to that of the $\langle 111\rangle$ [31]. The particles with $0.87<R<1.73$ have the $\{100\}$ and $\{111\}$ facets, which are named the truncated octahedral. Fig. 4 shows the correlativity of $R$ and the amount of $\mathrm{H}_{2} \mathrm{O}$, and the insets are their corresponding values and simulations. The result indicates that there is a 
linear correlativity between the truncated degrees and the amount of $\mathrm{H}_{2} \mathrm{O}$ [32]. The above results show that the exposed faces of $\left[\mathrm{Cu}_{3}(\mathrm{BTC})_{2}\right]_{n}$ are successfully tuned by adjusting the amount of water.

\section{Fabrication of hollow nanostructures}

Particularly, the successful fabrication of the hollow rough spheres (Figs 2e and f, type iv) inspired our interest considering the fact that hollow structured nanomaterials have shown intriguing properties and thus have wide use in many important fields $[33,34]$. In order to investigate the formation mechanism, time-dependent experiments were performed. As shown in Fig. S18, it is believed that an Os-

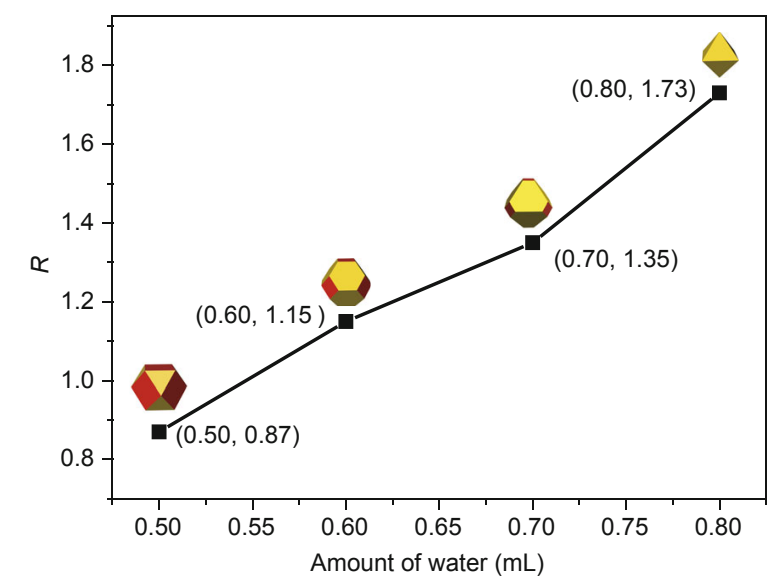

Figure 4 The correlativity of the truncated degree with the amount of $\mathrm{H}_{2} \mathrm{O}$. twald ripening [35] might govern this process. (See SI for detailed analysis)

Furthermore, other experiments were also carried out in an attempt to get more hollow structures. Intriguingly, increasing the temperature and reaction time to $140^{\circ} \mathrm{C}$ and $45 \mathrm{~min}$ with keeping the rest of the conditions for synthesizing solid octahedron (type i) unchanged, uniform partially hollow octahedrons (type $\mathrm{x}$ ) were obtained, as shown in Figs 5a-c. If the already-prepared $\left[\mathrm{Cu}_{3}(\mathrm{BTC})_{2}\right]_{n}$ octahedral crystals (type i) were dispersed in ethanol followed by sealing in a Teflon-lined autoclave and heating at $140^{\circ} \mathrm{C}$ for several hours, a gradual transformation from solid to hollow octahedrons occurred (Fig. S19). After 54 h, totally hollow octahedrons (type xi, Figs $5 \mathrm{~d}-\mathrm{f}$ ) were produced. The PXRD patterns of type $\mathrm{x}$ and vii prove them to be pure-phase $\left[\mathrm{Cu}_{3}(\mathrm{BTC})_{2}\right]_{n}$ (Fig. S20). The morphological transformation from the solid to hollow is also attributed to the Ostwald ripening mechanism $[17,36]$.

\section{Construction of noble metal@MOF nanocomposite and its catalytic property}

It is known that MOF nanostructures are excellent host to encapsulate nanoparticle catalysts to form core-shell nanostructures [37,38] and gas-involved reactions can be significantly enhanced by these nanocatalysts@MOF nanostructures $[17,18,38,39]$. Therefore, $\mathrm{Pd} @\left[\mathrm{Cu}_{3}(\mathrm{BTC})_{2}\right]_{n}$ nanostructures were prepared and applied in the reaction of selective hydrogenation of 1-chloro-2-nitrobenzene. As shown in Fig. 6, the addition of pre-synthesized $\mathrm{Pd}$ nanocubes [40] (Fig. S21) to the precursor solu-
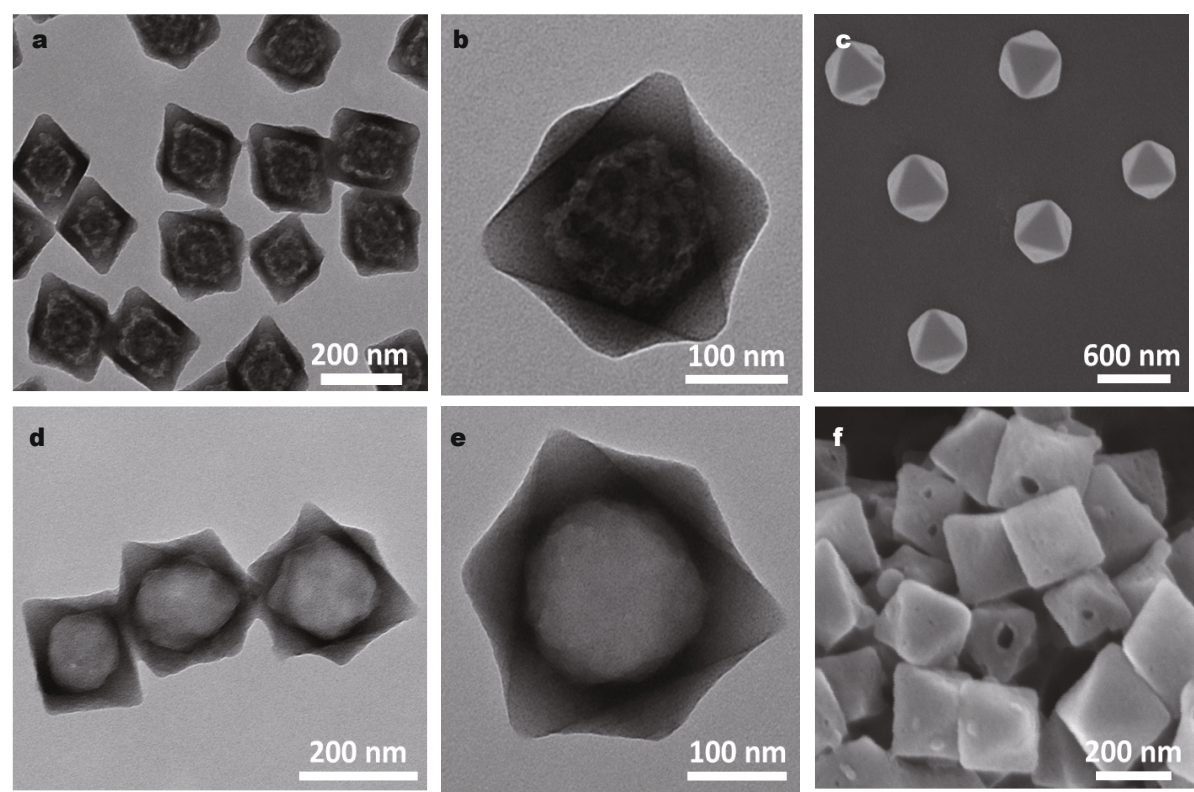

Figure 5 (a, b, d, e) TEM and (c, f) SEM images of $\left[\mathrm{Cu}_{3}(\mathrm{BTC})_{2}\right]_{n}$ crystals of $(\mathrm{a}-\mathrm{c})$ type $\mathrm{x},(\mathrm{d}-\mathrm{f})$ type $\mathrm{xi}$. 
a
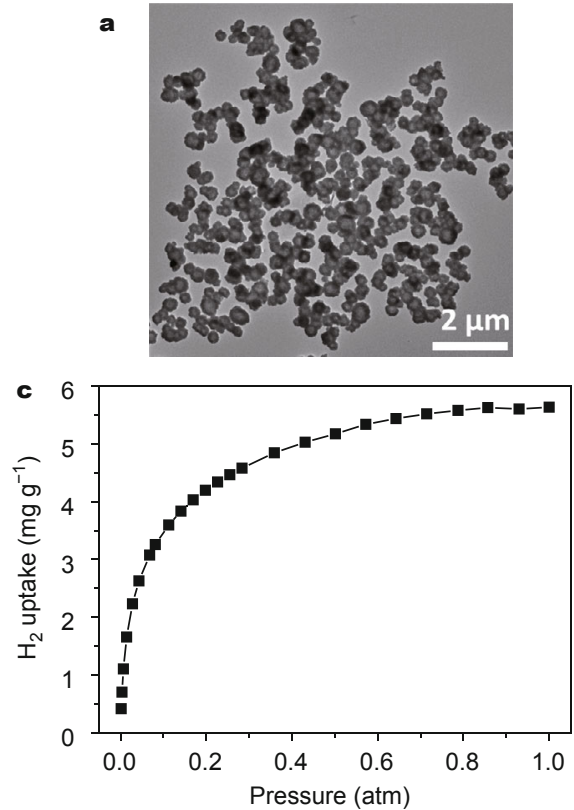

b

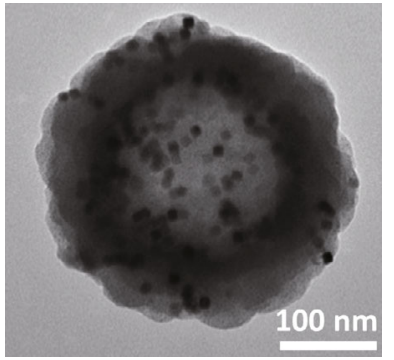

d

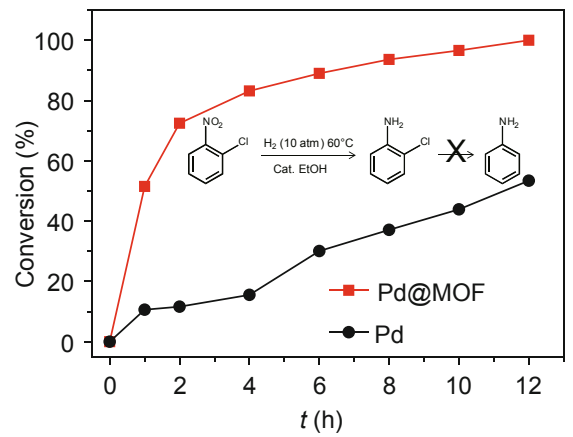

Figure 6 (a, b) TEM images of Pd@ $\left[\mathrm{Cu}_{3}(\mathrm{BTC})_{2}\right]_{n}$ nanostructures; (c) $\mathrm{H}_{2}$ sorption data for $\left[\mathrm{Cu}_{3}(\mathrm{BTC})_{2}\right]_{n} \mathrm{NCs}$ with low-pressure $\mathrm{H}_{2}(77 \mathrm{~K})$; (d) conversion (\%) as a function of time with Pd nanocubes and $\mathrm{Pd} @\left[\mathrm{Cu}_{3}(\mathrm{BTC})_{2}\right]_{n}$ nanostructures, the inset shows the catalytic reaction scheme of selective hydrogenation of 1-chloro-2-nitro-benzene.

tion of $\left[\mathrm{Cu}_{3}(\mathrm{BTC})_{2}\right]_{n}$ NCs leads to the formation of Pd@ $\left[\mathrm{Cu}_{3}(\mathrm{BTC})_{2}\right]_{n}$ nanostructures. The superior sorption ability of $\mathrm{H}_{2}$ was verified by the sorption data of $\mathrm{H}_{2}(5.63 \mathrm{mg}$ $\mathrm{g}^{-1}$ ) collected up to 1 bar at $77 \mathrm{~K}$. It was found that pure $\left[\mathrm{Cu}_{3}(\mathrm{BTC})_{2}\right]_{n}$ was inactive while Pd@ $\left[\mathrm{Cu}_{3}(\mathrm{BTC})_{2}\right]_{n}$ nanostructures exhibited much higher catalytic activity than pure Pd NCs. The maximum conversion of 1-chloro-2-nitrobenzene after $12 \mathrm{~h}$ was $53.42 \%$ using Pd nanocubes as catalyst, while for Pd@ $\left[\mathrm{Cu}_{3}(\mathrm{BTC})_{2}\right]_{n}$ nanostructures same conversion was obtained in just $1 \mathrm{~h}$. Thus it was verified that $\mathrm{H}_{2}$ storage in the $\left[\mathrm{Cu}_{3}(\mathrm{BTC})_{2}\right]_{n}$ might accelerate the catalytic process of Pd clusters (Fig. 6d). Moreover, structure of Pd@ $\left[\mathrm{Cu}_{3}(\mathrm{BTC})_{2}\right]_{n}$ nanostructures was retained even after the catalytic reaction (Fig. S22).

\section{CONCLUSION}

In summary, we suggested a simple method adopting POM anions to modulate the nucleation and growth processes and thus got various well-defined morphologies of $\left[\mathrm{Cu}_{3}(\mathrm{BTC})_{2}\right]_{n}$ crystals, such as octahedron, cubeoctahedron, truncated octahedron, hollow octahedron, hollow rough spheres and so on. POM anions prove to be efficient shape-directing agents. Considering the universality of the interactions and diversity of compositions and topologies of POMs, this strategy may be flexible and versatile. In fact, in addition to the example of HKUST-1 described in this context, uneven-surfaced and nanosheets-based hierarchical Fe/Co-BTC nanotubes were also successfully prepared employing Keggin-type POMs as modulator, which was described in another paper. The proposed strategy is expected to open up a new thought for rationally designing and synthesizing MOF NCs with specific shape.

Received 21 April 2015; accepted 12 May 2015;

published online 15 May 2015

1 Furukawa H, Cordova KE, O'Keeffe M, et al. The chemistry and applications of metal-organic frameworks. Science, 2013, 341: 1230444

2 Sumida K, Rogow DL, Mason JA, et al. Carbon dioxide capture in metal-organic frameworks. Chem Rev, 2011, 112: 724-781

3 Suh MP, Park HJ, Prasad TK, et al. Hydrogen storage in metalorganic frameworks. Chem Rev, 2011, 112: 782-835

4 Li JR, Sculley J, Zhou HC. Metal-organic frameworks for separations. Chem Rev, 2011, 112: 869-932

5 Yoon M, Srirambalaji R, Kim K. Homochiral metal-organic frameworks for asymmetric heterogeneous catalysis. Chem Rev, 2011, 112: 1196-1231

6 Cui Y, Yue Y, Qian G, et al. Luminescent functional metal-organic frameworks. Chem Rev, 2011, 112: 1126-1162

7 Li X, Wen J, Low J, et al. Design and fabrication of semiconductor photocatalyst for photocatalytic reduction of $\mathrm{CO}_{2}$ to solar fuel. Sci China Mater, 2014, 57: 70-100

8 Carné-Sánchez A, Imaz I, Cano-Sarabia M, et al. A spray-drying strategy for synthesis of nanoscale metal-organic frameworks and their assembly into hollow superstructures. Nat Chem, 2013, 5: 203-211

9 Flügel EA, Ranft A, Haase F, et al. Synthetic routes toward MOF nanomorphologies. J Mater Chem, 2012, 22: 10119-10133

10 Spokoyny AM, Kim D, Sumrein A, et al. Infinite coordination polymer nano- and microparticle structures. Chem Soc Rev, 2009, 38: 
1218-1227

11 Carné A, Carbonell C, Imaz I, et al. Nanoscale metal-organic materials. Chem Soc Rev, 2011, 40: 291-305

12 Zhou S, Zou X, Sun F, et al. Challenging fabrication of hollow ceramic fiber supported $\mathrm{Cu}_{3}(\mathrm{BTC})_{2}$ membrane for hydrogen separation. J Mater Chem, 2012, 22: 10322-10328

13 Bétard A, Fischer RA. Metal-organic framework thin films: from fundamentals to applications. Chem Rev, 2012, 112: 1055-1083

14 Horcajada P, Gref R, Baati T, et al. Metal-organic frameworks in biomedicine. Chem Rev, 2011, 112: 1232-1268

15 Horcajada P, Chalati T, Serre C, et al. Porous metal-organic-framework nanoscale carriers as a potential platform for drug delivery and imaging. Nat Mater, 2010, 9: 172-178

16 Taylor-Pashow KML, Rocca JD, Xie Z, et al. Postsynthetic modifications of iron-carboxylate nanoscale metal-organic frameworks for imaging and drug delivery. J Am Chem Soc, 2009, 131: 1426114263

17 Zhang Z, Chen Y, Xu X, et al. Well-defined metal-organic framework hollow nanocages. Angew Chem Int Ed, 2014, 53: 429-433

18 Zhang Z, Chen Y, He S, et al. Hierarchical Zn/Ni-MOF-2 nanosheetassembled hollow nanocubes for multicomponent catalytic reactions. Angew Chem Int Ed, 2014, 53: 12517-12521

19 Sindoro M, Yanai N, Jee AY, et al. Colloidal-sized metal-organic frameworks: synthesis and applications. Acc Chem Res, 2013, 47: 459-469

20 Stock N, Biswas S. Synthesis of metal-organic frameworks (MOFs): routes to various MOF topologies, morphologies, and composites. Chem Rev, 2012, 112: 933-969

21 Lin W, Rieter WJ, Taylor KML. Modular synthesis of functional nanoscale coordination polymers. Angew Chem Int Ed, 2009, 48: 650-658

22 Umemura A, Diring SP, Furukawa S, et al. Morphology design of porous coordination polymer crystals by coordination modulation. J Am Chem Soc, 2011, 133: 15506-15513

23 Liu W, Qu Y, Zhou W, et al. A versatile salicylic acid precursor method for preparing titanate microspheres. Sci China Mater, 2015, 58: 106-113

24 Long DL, Tsunashima R, Cronin L. Polyoxometalates: building blocks for functional nanoscale systems. Angew Chem Int Ed, 2010, 49: $1736-1758$

25 Yin P, Li T, Forgan RS, et al. Exploring the programmable assembly of a polyoxometalate-organic hybrid via metal ion coordination. J Am Chem Soc, 2013, 135: 13425-13432

26 Han XB, Zhang ZM, Zhang T, et al. Polyoxometalate-based cobalt-phosphate molecular catalysts for visible light-driven water oxidation. J Am Chem Soc, 2014, 136: 5359-5366

27 Yu R, Kuang XF, Wu XY, et al. Stabilization and immobilization of polyoxometalates in porous coordination polymers through hostguest interactions. Coord Chem Rev, 2009, 253: 2872-2890

28 Chui SSY, Lo SMF, Charmant JPH, et al. A chemically functionalizable nanoporous material $\left[\mathrm{Cu}_{3}(\mathrm{TMA})_{2}\left(\mathrm{H}_{2} \mathrm{O}\right)_{3}\right]_{n}$. Science, 1999,
283: $1148-1150$

29 Bajpe SR, Kirschhock CE , Aerts A, et al. Direct observation of molecular-level template action leading to self-assembly of a porous framework. Chem Eur J, 2010, 16: 3926-3932

30 Kang L, Wang Z, Cao Z, et al. Colloid chemical reaction route to the preparation of nearly monodispersed perylene nanoparticles: size-tunable synthesis and three-dimensional self-organization. J Am Chem Soc, 2007, 129: 7305-7312

31 Wang ZL. Transmission electron microscopy of shape-controlled nanocrystals and their assemblies. J Phys Chem B, 2000, 104: 1153 1175

32 Xiang G, Zhuang J, Wang X. Morphology-controlled synthesis of inorganic nanocrystals via surface reconstruction of nuclei. Inorg Chem, 2009, 48: 10222-10230

33 Lou XW, Archer LA, Yang Z. Hollow micro-/nanostructures: synthesis and applications. Adv Mater, 2008, 20: 3987-4019

34 Zhao Y, Lin LN, Lu Y, et al. Templating synthesis of preloaded doxorubicin in hollow mesoporous silica nanospheres for biomedical applications. Adv Mater, 2010, 22: 5255-5259

35 Chun $\mathrm{ZH}$. Ostwald ripening: a synthetic approach for hollow nanomaterials. Curr Nanosci, 2007, 3: 177-181

36 Huo J, Wang L, Irran E, et al. Hollow ferrocenyl coordination polymer microspheres with micropores in shells prepared by ostwald ripening. Angew Chem Int Ed, 2010, 49: 9237-9241

$37 \mathrm{Hu} \mathrm{P}$, Morabito JV, Tsung CK. Core-shell catalysts of metal nanoparticle core and metal-organic framework shell. ACS Catal, 2014, 4: 4409-4419

$38 \mathrm{Li}$, Hu J, Deng M, et al. Integration of an inorganic semiconductor with a metal-organic framework: a platform for enhanced gaseous photocatalytic reactions. Adv Mater, 2014, 26: 4783-4788

39 Xu X, Zhang Z, Wang X. Well-defined metal-organic-framework hollow nanostructures for gas-involved catalytic reactions. Adv Mater, doi: 10.1002/adma. 201500789

$40 \mathrm{Xu} \mathrm{B}$, Zhang Z, Wang X. Formamide: an efficient solvent to synthesize water-soluble and sub-ten-nanometer nanocrystals. Nanoscale, 2013, 5: 4495-4505

Acknowledgements This work was supported by the National Natural Science Foundation of China (21431003, 91127040 and 21221062), and the State Key Project of Fundamental Research for Nanoscience and Nanotechnology (2011CB932402).

Author contributions Wang $X$ designed this study and supervised the experimental work. Xu X, Lu Y, Yang Y and Nosheen F performed the synthesis and characterizations. All authors contributed to the general discussion and wrote the manuscript together.

Conflict of Interest The authors declare that they have no conflict of interest.

Supplementary information Experimental details and supplementary data are available in the online version of the paper. 


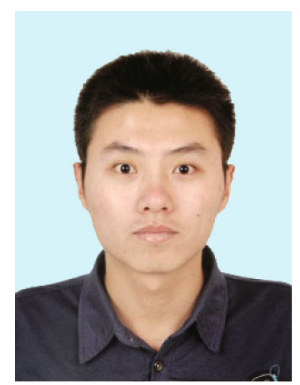

Xu Xiaobin was born in 1990. He received his BSc degree from the Faculty of Chemistry, Northeast Normal University (NENU) in 2012. Then he joined the Department of Chemistry at Tsinghua University and conducted research under the supervision of Prof. Xun Wang. His research interests include synthesis, self-assembly and application of polyoxometalates and metal-organic frameworks nanomaterials.

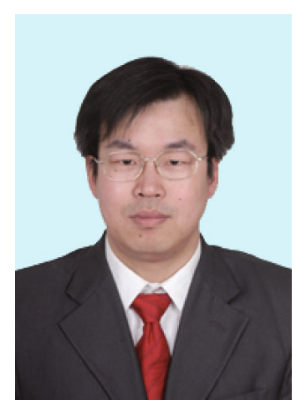

Wang Xun was born in 1976. He received his PhD degree in chemistry from the Department of Chemistry, Tsinghua University in 2004. Currently, he is a professor at the Department of Chemistry, Tsinghua University. His research interests include synthesis, assembly, characterization and application of ultrathin nanomaterials. His main awards include the Finalist for the IUMRS-MRS Singapore Young Researchers Award (2014), the Science and Technology Award for Chinese Youth (2009), the National Fund for Outstanding Young Scientists (2007) and the IUPAC Prize for Young Chemists (2005).

中文摘要 在金属阳离子和多酸阴离子之间广泛存在着一种超分子相互作用, 它经常被用来合成多酸基金属-有机骨架晶态材料. 这 种相互作用还可以用来调控金属-有机骨架材料的成核生长过程, 从而实现其纳米晶体的可控制备. 本文将Keggin型多酸阴离子引入到 金属-有机骨架材料的前驱体溶液中作为调节剂, 得到一系列形貌规则、尺寸均一的 $\left[\mathrm{Cu}_{3}(\mathrm{BTC})_{2}\right]_{n}(\mathrm{BTC}=1,3,5$-苯三甲酸)纳米晶, 包括 八面体、截角八面体、多级空心球、空心八面体等. 此外, 这种体系可以用于构建贵金属纳米晶@金属-有机骨架复合材料, 其催化性 质相对于单纯的贵金属纳米晶有较大提高. 